\title{
THE ROLE OF NUTRITION IN BRAIN DEVELOPMENT
}

\author{
Dana-Teodora Anton-Paduraru, Ana Simona Drochioi, Oana Teslariu \\ $3^{\text {rd }}$ Clinic of Pediatrics, "Gr. T. Popa” University of Medicine and Pharmacy, Iasi
}

\begin{abstract}
The brain is an organ that functions harmoniously special only through a balanced intake of biologically active substances. The phrase "food for brain" expresses the important role of nutrition in the development and energy supply of the brain. There is a brief presentation of data on the development of the brain, the existence and functionality of the microbiome-gut-brain axis and the impact of the key nutrients in these complex processes. The authors concluded that nutritional assistance to the pregnant woman since the onset of pregnancy, the prophylaxis of prematurity, the early prevention of the gut microbiome of infant through encouragement of natural birth and breast-feeding are necessary conditions for the development of the brain.
\end{abstract}

Keywords: brain, nutrition, importance

\section{INTRODUCTION}

The brain is a special organ whose functionality depends on the generation of electrical potentials, and for exercising its special functions it needs an increased amount of nutrients (folic acid, iron, zinc, choline, fatty acids). The brain works harmoniously only through a balanced intake of biologically active substances such as glucose, unsaturated fatty acids, phospholipids, vitamins and minerals (1).

Nutrition is an environmental factor that can modulate the expression of genetic factors. Moreover, nutrition can have direct effects on the gene expression in the brain. The phrase "brain food" expresses the important role of nutrition in the development and energy supply of the brain.

\section{BRAIN DEVELOPMENT}

In early fetal and postnatal period, some regions of the brain (the hippocampus, the visual and auditory cortex, the striatum) develop rapidly, and they become functional due to morphogenesis and synaptogenesis. Twenty-two days after conception the neural tube starts forming, and some nutrients (folic acid, copper, vitamin A) are needed already. In the $2^{\text {nd }}-3^{\text {rd }}$ quarters of the pregnancy important processes of growth and maturation of the brain take place: both the white and gray matter increase their volume, the axons, the glial cells, the oligodendro- cytes and neurons develop quickly, and between 24-40 weeks of pregnancy the axons build connections. Neurons proliferate and migrate into the cerebral cortex and the nuclear structures of the grey substance (2-4).

Premature birth happens when the brain is in a critical period of development, in particular the white structures, making it susceptible to aggressions that are in turn an important cause of neurodevelopmental disorders (3).

\section{Microbiome-gut-brain axis}

The interaction between the brain and the gut is well known, but in recent years the importance of microbiome has been highlighted in this two-way communication, thus defending the concept of "microbiome-gut-brain" axis. The mechanisms by which the intestinal microbiome communicates with the brain are not fully deciphered, as they include immunological, endocrine and nervous pathways.

The gut microbiome has the ability to modulate the brain development and the behavior. Immune cells, cytokines and chemokines mediate the mechanisms by which the intestinal microbiome may interact with the brain. Conversely, the brain can affect the small intestine via neurotransmitters that have an impact on the immune function and by altering the level of cortisol, the intestinal motility and permeability, Fig. 1 (3).

Corresponding author:

Ana Simona Drochioi. "Gr. T. Popa” University of Medicine and Pharmacy, 16 Universitatii Street, Iasi

E-mail: simonadrochioi@yahoo.com 


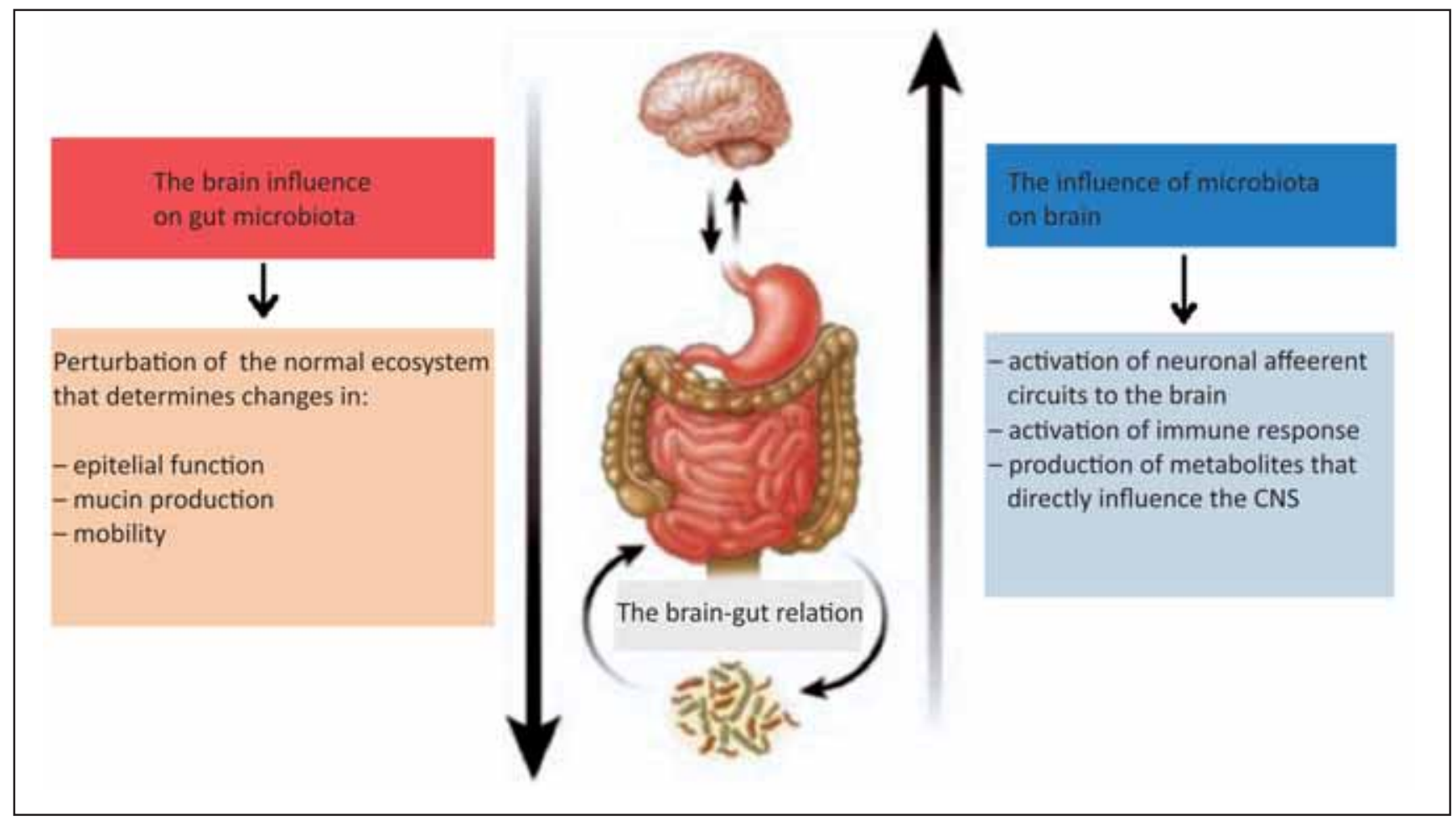

FIGURE 1. Microbiome-gut-brain axis (after Keunen-3)

Legend: CNS-central nervous system

\section{THE ROLE OF NUTRITION IN BRAIN DEVELOPMENT}

Adequate nutrition during pregnancy and the first two years of life is required for the normal development of the brain.

Many mothers and children from both underdeveloped countries and the developing countries are at risk for malnutrition. About 200 million children aged under 5 years in these countries have delays in cognitive and motor development and socio-emotional skills impairment $(4,5)$.

Fetal protein-caloric malnutrition occurs as a result of intrauterine growth delay and it is caused by severe malnutrition and maternal hypertension. The intrauterine growth delay, severe or prolonged, leads to an impaired fetal growth of the head along with an impaired growth of the whole body. Even in the absence of microcephaly, infants with intrauterine growth delay have, in $15 \%$ of cases, slight anomalies of development characterized by cognitive changes. Post-birth malnutrition usually occurs in premature babies who do not get enough liquids or do not tolerate high quantities of nutrients (5).

Early acute malnutrition has persisting consequences on the development of the brain even after the nutritional rehabilitation. Some studies have compared children of school age with a history of an episode of severe acute malnutrition to a control group or siblings who did not have malnutrition, concluding that those in the first category had a lower intelligence quotient (IQ), cognitive disorders and weaker school performance. Also, chronic malnutrition is associated with delay in cognitive and motor development. (5).

The global and specific effects of malnutrition on the development of the brain depend on the time that the nutrient deficiency acts, the severity of the deficit and the possibility of recovering the deficit. Some nutrients that are useful in the development of the brain at a certain time can be toxic in another moment during the development (4).

The nutrients regulate the brain development during the fetal and postnatal period. Between the weeks 24-45 of pregnancy, brain development is vulnerable to the nutritional deficits due to the neurologic processes that include the rapid formation of synapses and myelination.

Nutrients are needed not only for neurons, but also for the glial cells. For some areas, early nutritional deficits affect the cellular proliferation, their number being affected. The late nutritional damage influences the differentiation, including the size, the complexity, the synaptogenesis and the dendritic arborization. They can also have effects not only on the neuroanatomy, but also on the neurochemistry and neurophysiology. The neurochemical changes include changes in the synthesis of neu- 
rotransmitters, the synthesis of receptors and the recapture of neurotransmitters (3-5).

Brain development depends mostly on the nutrient intake in the period between the second quarter of pregnancy and the age of 2. Malnourished children, who are deprived of calories and protein during that period, do not develop neither physically nor mentally. Their brain is smaller than normal due to the discontinued increase in dendrites, myelination, and a smaller number of glial cells. Inadequate brain growth explains why malnourished children in fetal or infant period show cognitive and behavioral deficits including language delay, motor retardation, low IQ and low school performance (3-5).

The birth weight and the size of the brain of the child depends on the quality of his/her mother's nutrition during pregnancy. To ensure a proper fetal growth, the pregnant woman should increase in weight by approximately $20 \%$ of ideal pre-pregnancy weight. It involves the consumption of 300 $\mathrm{kcal} /$ day, including 10-12 $\mathrm{g}$ protein.

After birth, brain growth depends on the quality of child's nutrition. It has been shown that iron deficiency determines the cognitive deficits in young children; iron is necessary to maintain a suitable number of RBCs, conveyors of oxygen. Infants fed artificially require iron supplemented formulas.

Due to fast myelination, children require a high level of fat in the diet until the age of 2 years $(50 \%$ of total calories), provided by human milk or formula milk in their first year of life. In the second year of life, cow's milk also can be a good source of fat and protein. After the age of 2, children should receive a diet in which fat ensures a maximum of $30 \%$ of the total calories (5-7).

\section{Main nutrients involved in brain development}

All nutrients are required for nerve cell development, but some of them like protein, iron, zinc, iodine, selenium, vitamin A, folates, choline, longchain polyunsaturated fatty acids - LCPUFA - have stronger effects in the late fetal and neonatal period.

\section{Iron}

Iron is rapidly absorbed by the fetus in the last trimester of the pregnancy and it is necessary for the basic neural processes such as myelination, production neurotransmitters and energy metabolism. The iron status in newborn babies may be affected as a result of severe maternal iron-deficiency anemia, the increase of the quantity of iron needed for erythropoiesis in case of maternal diabetes or insufficient deposit due to premature birth. The fetal and neonatal iron deficiency leads to a reduction of oxidative metabolism in the hippocampus and frontal cortex, high intracellular concentrations of neuronal glutamate, low concentrations of dopamine, and altered profiles of the fatty acid and myelin (2).

Some studies have shown that low levels of ferritin in the umbilical cord blood are associated to the low neurological development during school age. Also, the infants with iron-deficiency anemia from diabetic mothers have memory impairments, and the premature babies have abnormal reflexes 36 weeks after conception. Longitudinal studies have shown that children who had iron deficiency anemia in the infant period continued to have a lower IQ, social problems and attention deficit disorders in adolescence $(5,6)$.

\section{Zinc}

Zinc ranks $4^{\text {th }}$ in terms of the concentration in the brain, helping the structure and functioning of the brain and it is involved in many biological processes that affect brain development, including DNA and RNA synthesis, metabolism of proteins, carbohydrates and fats.

Zinc is essential in the pre- and postnatal period for growth and maturation. In the first weeks of pregnancy zinc is essential for cell multiplication, embryo implantation, cell differentiation and maturation of organs. In the last few weeks of pregnancy, zinc deficiency prevents the neuronal migration and replication. The low maternal zinc intake during pregnancy and lactation are associated with attention disorders and motor function alteration at the age of 6 months (9). At neuronal level, the function of the pre-synaptic buttons depends on the zinc level. The fetal zinc deficiency determines the decrease of DNA, RNA and proteins in the brain. Experimentally it has been shown that zinc is important for the development of the medial temporal lobe, frontal lobe and cerebellum (2).

Fetuses from zinc-deficient mothers have reduced fetal movements and variable cardiac frequency, which suggest the impaired stability of the autonomous nervous system. Low zinc levels associated with a modified zinc/copper ratio correlate with the severity of symptoms associated with autism.

Premature infants may present a greater risk to have zinc deficiency because $60 \%$ of the fetal zinc is provided in the last trimester of pregnancy. Friel et al. quoted by Mathur and Kagarwal followed the neurological development of 52 premature babies using the Griffith's scale and they have noticed that the motor development was greater in those who were supplemented with zinc compared to the pla- 
cebo. Zinc supplementation by the corrected age of 3 months of the premature babies fed naturally improved the attention and decreased the signs of hyperexcitability and reflexes (10).

\section{Copper}

Copper is a bivalent action involved in the energy metabolism of the brain, in dopamine metabolism, in the antioxidant processes and in the absorption of iron. The development of the cerebellum seems mostly affected by the copper deficiency, with long-term effects on the motor function, balance and coordination $(8,11,12)$.

\section{Iodine}

Iodine is necessary for the synthesis of thyroid hormones that are essential in the development of the central nervous system, including the neurogenesis, the neuron migration, the growth of dendrites, the synaptogenesis and the myelination. The severe deficiency of iodine before and during pregnancy may lead to low production of thyroid hormones in the mother and cretinism in the child. Even in the absence of cretinism, the chronic iodine deficit adversely affect intelligence (IQ lower by 13.5 points) $(13,14)$.

\section{Essential fatty acids}

Essential fatty acids and their derivatives (Docosahexaenoic acid-DHA and arachidonic acid (ARA)-) are part of the structure of the brain tissue, including of the cellular membrane.

The effect of essential fatty acids during pregnancy is not clarified. Fatty acids are important for fetal development of neurons, but randomized trials on the supplementation of the mother with essential fatty acids have provided mixed results. Long-chain polyunsaturated fatty acids (LCPUFA) in particular, the docosahexaenoic acid, (DHA) are neurobiological agents involved in numerous neuronal processes; the lack of these acids affects the neurogenesis, the structure and integrity of the neuronal membrane, the synaptogenesis and the myelination $(2,15)$. Maternal supplementation with DHA during pregnancy has neuroprotective effects against prenatal brain dysfunction induced by ischemic dysfunction due to stress and hypoxia (15, 16). In premature babies, drinking milk formula with omega 3 - type LCPUFA improves the visual function and the cognitive development.

\section{Other nutrients}

Selenium: the effects are mediated through thyroid hormone metabolism. Selenium deficiency correlates with a decrease of the cognitive function and the impairment of the motor function. The dietary intake of Selenium is important in the synthe- sis of selenoprotein necessary for the proper functioning of the brain (15-17).

Folates and choline: the effects are mediated via the carbon metabolism, DNA methylation and neurotransmitters synthesis.

Folates are effective in the synthesis of nucleotides, in DNA integrity and phospholipid methylation in the neuronal membrane. Elevated maternal concentrations of folates during pregnancy can influence the cognitive development of the child (16). The importance of the folates for the brain development is evidenced by the protective effect of the supplementation versus the malformations of the neural tube (spina bifida), which was first suggested more than 50 years ago. Current recommendations aim at the day-to-day administration to all women of reproductive age of a quantity of $0.4 \mathrm{mg}$ of folic acid along with a diet rich in folates. The proportion of the neural tube defects, which can thus be prevented, is not well determined, but it probably lies between 50-60\% (18).

Vitamin A plays a role in setting up the connections between the brain and the sense organs. Vitamin A deficiency is a neuro teratogen risk factor in preconception period, but in the post-natal period, it is associated with neuronal and retinal degeneration (7).

Vitamins of the $B$ group contribute to the optimal operation of CNS due to their role as cofactor in many catalysing reactions, which are necessary for the synthesis and functioning of neurotransmitters and for myelination.

Vitamin B6 is a co-factor in various enzymatic reactions in the CNS, which catalyses the metabolism of amino acids and neurotransmitters. It is necessary in the cognitive development and the development of short and long-term memory. Vitamin B6 deficiency can cause seizures along with abnormalities in the structure of the brain and psychomotor retardation (19). Vitamin B12 is a key nutrient for the development and functioning of the brain. It is involved in maintaining the balance between the neurotrophic and the neurotoxic factors, because the neurotrophins affect the proliferation, differentiation and survival of neurons. Animal studies have shown that supplementation with Vitamin B12 is capable to maintain the level of DHA and of the neurotrophic factor in the cortex and hippocampus $(15,20)$.

Probiotics: the microorganisms that colonize the intestine may be potential neuroprotectors agents especially for premature babies. They may be beneficial for the brain development in premature babies due to the favorable influence on the 
immune response, leading to lower inflammation, which, in consequence, can alleviate the lesions of the white matter. The administration of probiotics can alter the composition of the intestinal microbiome and their ability to modulate the microbiomegut-brain axis depends on the strain. In adults it has been observed that a mixture of probiotics containing Lactobacillus helveticus and Bifidobacterium longum induce beneficial psychological effects and lowers cortisol, and the same combination reduces anxiety in mice. The combination between Bifidobacterium longum and Lactobacillus helveticus reduces anxiety significantly. Animal studies showed that Lactobacillus rhamnosus (which exerts effect through the vagus nerve) attenuates the symptoms of depression and anxiety. The Lactobacillus helveticus NS8 strain decreases neuro-inflammation, reduces the serotonin metabolism, decreases anxiety and restores the cognitive function. Bifidobacterium infantis lowers the level of serotonin metabolites in the prefrontal cortex and of the metabolites of dopamine in the amygdaloidal cortex, demonstrating that it can promote the anti-depressant effect as a result of the modulation of mono amines (serotonin and dopamine) (3).

The role of natural nutrition in brain development: human milk provides the best mix of nutri-

\section{REFERENCES}

1. Rosales F. Understanding the Role of Nutrition in the Brain \& Behavioral Development of Toddlers and Preschool Children: Identifying and Overcoming Methodological Barriers. Nutr Neurosci 2009; 12(5):190-202

2. Georgieff M. Nutrition and the developing brain: nutrient priorities and measurement. Am J Clin Nutr 2007; 85(suppl), 61:4S-20S.

3. Kristin K., VanElburg R., Van Bel F., Benders M. Impact of nutrition on brain development and its neuroprotective implications following preterm birth. Pediatric Research 2015; 77,1:148-155.

4. Elizabeth P., Dewey K. Nutrition and brain development in early life. Nutrition Reviews 2014; 72(4):267-284.

5. Elizabeth P., Dewey K. Nutrition and brain development in early life. A\&T Technical Brief 2012; 4:1-13.

6. Aida M.S. Nutrition and brain development. SA Fam Pract 2007; 49(3):40-42.

7. Anett N., Li Jianghong, Siobhan H., Foster J., Oddy W. The role of nutrition in children's neurocognitive development, from pregnancy through childhood. Front. Hum. Neurosci. http://dx.doi.org/10.3389/ fnhum.2013.00097

8. Scheiber I., Mercer J., Dringen R. Metabolism and functions of copper in brain. Progress in Neurobiology 2014; 116:33-57 doi: 10.1016/j.pneurobio.2014.01.002.

9. Sandstead H. Zinc is essential for brain development and function. The Journal of Trace Elements in Experimental Medicine 2003; 16(4):165-173

10. Mathur N.B., Kagarwal D. Zinc Supplementation in Preterm Neonates and Neurological Development: A Randomized Controlled Trial. INDIAN PEDIATRICS 2015; 52:951-955.

11. Johnson T. Copper and brain function.http://naldc.nal.usda.gov/ download/46926/PDF ents necessary for brain development. Due to its composition, which includes growth factors, hormones, DHA and choline, human milk may improve cognitive development. In the meantime, the physical act of breastfeeding may improve the relationship between mother and infant, being important for the cognitive and socio-emotional development.

Studies on school-age children from developed countries who were breastfed during infancy showed that they had a higher intelligence quotient compared to children who have been fed artificially $(3,4)$.

\section{CONCLUSIONS}

Nutrition is one of the factors that may affect the development and operation of the brain, and diet can affect the cognitive development in different stages of life (fetus, newborn, infant, child).

Nutritional assistance to the pregnant woman since the onset of pregnancy, the prophylaxis of prematurity, the early prevention of the gut microbiome of infant through encouragement of natural birth and breast-feeding are necessary conditions for the development of the brain.

12. Manto M. Abnormal Copper Homeostasis: Mechanisms and Roles in Neurodegeneration. Toxics 2014, 2, 327-345; doi:10.3390/toxics2020327.

13. Lozoff B., Georgieff M. Iron Deficiency and Brain Development. Seminars in Pediatric Neurology 2006; 13(3):158-165.

14. Zimmermann $\mathbf{M}$. The role of iodine in human growth and development. Seminars in Cell \& Developmental Biology 2011; 22: 645-652.

15. Richa R., Anvita K., Sadhana J. Novel insights into the effect of vitamin B12 and omega-3 fatty acids on brain function. Journal of Biomedical Science (2016) 23:17 DOI 10.1186/s12929-016-0241-8.

16. Van deRest O., VanHooijdonk L., Esmee D., Olga S., Ans E., deGroot L. B Vitamins and n-3 Fatty Acids for Brain Development and Function: Review of Human Studies. Ann Nutr Metab 2012; 60:272-292.

17. Pillai R., Jane U.K., Bellinger F. Selenium and Selenoprotein Function in Brain Disorders. International Union of Biochemistry and Molecular Biology 2014; 66(4):229-239.

18. Pitkin R. Folate and neural tube defects. Am J Clin Nutr 2007; 85(suppl):285S-8S.

19. Monique A., Floris G., Van der Ham Maria, De Koning T., Marjolein B., Visser W. şi col. Vitamin B6 Vitamer Concentrations in Cerebrospinal Fluid Differ Between Preterm and Term Newborn Infants. PEDIATRICS 2012; 130(1):191-198.

20. Maureen B. Effects of vitamin B12 and folate deficiency on brain development in children. Food Nutr Bull. 2008 June; 29(2 Suppl): S126-S131. 